\title{
Uso de resíduo do beneficiamento de um pegmatito com albita e espodumênio no desenvolvimento de fritas e esmaltes cerâmicos
}

\section{(Use of waste from mineral processing of a pegmatite with albite and spodumene in the development of frits and ceramic glazes)}

\author{
J. L. Coelho, H. C. M. Lengler, S. R. Bragança \\ Universidade Federal do Rio Grande do Sul - DEMAT, Av. Osvaldo Aranha 99/711, Porto Alegre, RS, 90035-190 \\ saulorb@ufrgs.br
}

\begin{abstract}
Resumo
O uso de resíduos incorporados a massas cerâmicas tornou-se comum nas últimas décadas como meio de se reduzir os custos de produção e preservar os recursos minerais. O presente trabalho teve como objetivo avaliar o uso de resíduo do beneficiamento de uma rocha, o qual é composto por quartzo, albita e espodumênio, para a produção de esmaltes e fritas cerâmicas, a serem utilizados na cerâmica tradicional. Esses produtos foram caracterizados quanto à composição química, fusão e expansão térmica. Em uma composição comercial de frita transparente, foram adicionadas quantidades crescentes do resíduo (7 a 30\%), avaliando-se a fusão das novas composições. Estas foram testadas em uma formulação de esmalte, avaliando-se a expansão térmica, os defeitos de queima e a estética do produto final (transparência, brilho, ausência de bolhas e cristais insolúveis). Na segunda etapa, foi desenvolvido um esmalte por meio da adição do resíduo em uma formulação de esmalte comercial mate, avaliando-se o brilho, a cobertura (opacidade) e o desenvolvimento de cor. Os resultados obtidos demonstraram a redução da expansão térmica e da temperatura de amolecimento do esmalte formulado com a frita desenvolvida. Por meio de ajustes na composição química, como a redução de óxidos alcalinos e aumento de alcalinos terrosos, foi possível obter formulações com as propriedades de produtos aceitos no mercado, com as características técnicas e estéticas desejadas. O óxido de lítio atuou como fluxante nas novas formulações, sem alterar significativamente as propriedades finais. Adicionalmente, obteve-se a redução na quantidade de corantes no esmalte desenvolvido, o que permite a diminuição do custo associado às matérias-primas.

Palavras-chave: resíduos, fritas, esmalte, expansão térmica, propriedades técnicas.
\end{abstract}

Abstract

The use of waste incorporated into the ceramic bodies has become common in recent decades as a means to reduce production costs and to preserve mineral resources. This study aimed to evaluate the use of waste from the mineral processing of a rock. The waste composed of quartz, albite and spodumene was used to produce frits and ceramic glazes to traditional ceramics. These materials were characterized by chemical composition, fusion, and thermal expansion. In a commercial transparent frit composition, increasing percentage of the residue ( 7 to 30\%) was added, and then the fusion of the new compositions was evaluated. With these frits, a ceramic glaze formulation was developed, evaluating the thermal expansion coefficient, firing defects, and aesthetics of the final product (transparency, gloss, no bubbles, and insoluble crystals). In the second step, a ceramic glaze was developed, with the addition of residue on a commercial matte glaze formulation, and evaluating the gloss, opacity and color development. The results showed reduction in the thermal expansion and softening temperature of the developed glaze. By adjusting the chemical composition, decreasing alkaline oxides and increasing alkaline earth oxides, it was possible to prepare formulations with product properties widely accepted in the market, with the technical and desired aesthetic characteristics. The lithium oxide acted as a flux in the new formulations, with little influence on the final properties. Additionally, there was the reduction of the colorant amount, decreasing the cost associated with raw materials.

Keywords: wastes, frits, ceramic glaze, thermal expansion, technical properties.

\section{INTRODUÇÃO}

Nas últimas décadas, a avaliação das questões ambientais tornou-se parte importante do processo de produção da indústria, procurando-se o emprego de processos mais eficientes, com a redução do consumo de energia, diminuição do efluente gerado e a otimização do uso de matériasprimas. Uma maneira de se alcançar este objetivo, como demonstram diversos estudos, é o reaproveitamento de resíduos industriais ou resíduos oriundos do beneficiamento mineral, propiciando-se uma economia das matérias-primas naturais e, por conseguinte, o aumento da preservação dos recursos naturais. A utilização de resíduos pela indústria cerâmica é geralmente viabilizada através da substituição parcial das matérias-primas na composição das massas cerâmicas, desde que não se comprometa as propriedades e especificações técnicas dos produtos finais. A maior parte dos estudos técnicos sobre o assunto demonstra 
a utilização de diferentes resíduos em formulações de produtos da cerâmica vermelha [1]. Por vezes, encontra-se a utilização na produção de grés, porcelanas e refratários [2-5]. No entanto, poucos trabalhos apresentam o uso de resíduos e materiais reciclados na composição de produtos como fritas e esmaltes, possivelmente, devido a maior dificuldade e complexidade de se formular e ajustar as propriedades técnicas desses materiais. Estes, embora utilizem uma quantidade relativamente menor de insumos minerais, apresentam maior valor agregado. Portanto, o desenvolvimento de metodologia para o emprego de resíduos à formulação desses produtos, significa propiciar à indústria de esmaltes e fritas uma oportunidade de tornar seu processo ecologicamente mais correto.

Como reportado, encontram-se poucos exemplos de aproveitamento de resíduos em fritas e esmaltes na literatura. Um trabalho mostra a adição de diferentes resíduos (vidro sodo-cálcico, granito e cal-xisto) na formulação de esmaltes para revestimentos cerâmicos, alcançando-se parâmetros de expansão térmica, dureza e temperatura de acoplamento compatíveis com os de esmaltes comerciais [6]. O resíduo refratário de moldes de fundição, ricos em $\mathrm{Al}_{2} \mathrm{O}_{3}$ e $\mathrm{SiO}_{2}$, foi usado na formulação de fritas, obtendose um baixo custo do produto [7]. O emprego de matériasprimas que contém lítio em sua composição vem crescendo na última década [8-10], sendo utilizada, principalmente, a forma mineral (espodumênio), a partir de rochas como pegmatitos graníticos, ou a forma sintética (carbonato de lítio), esta, porém, de maior custo [11]. O objetivo do presente trabalho foi avaliar o uso de um resíduo (contendo lítio) do beneficiamento mineral de uma rocha, como uma matéria-prima para o desenvolvimento de composições de fritas e de esmaltes cerâmicos. A avaliação da frita foi realizada inicialmente por meio de testes de fusão e na composição de um esmalte, caracterizando-se a expansão térmica e avaliando-se as propriedades físicas e estéticas. $\mathrm{Na}$ segunda parte do trabalho, foi desenvolvido um esmalte por meio de mistura do resíduo em uma formulação de esmalte comercial, e foram avaliados os aspectos visuais (textura, brilho, cobertura e desenvolvimento de cor) e a expansão térmica.

\section{MATERIAIS E MÉTODOS}

O resíduo do beneficiamento da rocha com espodumênio (RSL) foi estudado em composições de frita e de esmalte, procurando-se avaliar também o efeito da presença de lítio nas formulações. Esse resíduo foi fornecido pela empresa Colorminas - Colorifício e Mineração S.A., sendo um subproduto da mineração de pegmatitos de Minas Gerais. A caracterização completa desse material, incluindo o estudo petrográfico da rocha mãe, pode ser encontrada em trabalho anterior $[10,12]$. Parte da metodologia utilizada no presente estudo é a mesma empregada na referida indústria, no desenvolvimento de seus produtos.

A composição química do resíduo RSL determinada por espectroscopia de fluorescência de raios X (WDS Philips PW
2400) é mostrada na Tabela I (a determinação do óxido de lítio foi realizada por espectroscopia de absorção atômica), a qual apresenta também a composição da frita fornecida pelo fabricante. As composições utilizadas no estudo das fritas são apresentadas na Tabela II. As fases cristalinas determinadas por difratometria de raios X (Bruker, D2 phaser) são apresentadas na Fig. 1 (DRX-JCPDS/número do arquivo: espodumênio 33-0786; albita 01-07390 e quartzo 05-490). Como mostra a Fig. 1, o RSL apresenta como principais fases cristalinas o espodumênio e a albita, os quais atuam como fluxantes, e o quartzo, o qual atua como formador de fase vítrea e, principalmente, aumenta o coeficiente de expansão térmica em formulações da cerâmica tradicional [11]. O pequeno pico no difratograma foi referido apenas como alumino-silicato (Fig. 1), pois sua identificação é difícil de precisar, provavelmente, em consequência da moagem e devido à pequena quantidade do mesmo.

Tabela I - Análise química do RSL e da frita STD (\% em massa).

[Table I - Chemical composition of RSL and STD frit (weight $\%)$.

\begin{tabular}{|c|c|c|}
\hline Óxidos (\%) & RSL & Frita transparente (STD) \\
\hline $\mathrm{SiO}_{2}$ & 77,15 & 63,56 \\
\hline $\mathrm{Al}_{2} \mathrm{O}_{3}$ & 15,26 & 8,74 \\
\hline $\mathrm{Na}_{2} \mathrm{O}$ & 3,26 & $3,87\left(\mathrm{Na}_{2} \mathrm{O}+\mathrm{K}_{2} \mathrm{O}\right)$ \\
\hline $\mathrm{K}_{2} \mathrm{O}$ & 1,79 & - \\
\hline * $\mathrm{B}_{2} \mathrm{O}_{3}$ ou $\mathrm{Li}_{2} \mathrm{O}$ & $1,59\left(\mathrm{Li}_{2} \mathrm{O}\right)$ & $5,90\left(\mathrm{~B}_{2} \mathrm{O}_{3}\right)$ \\
\hline $\mathrm{Fe}_{2} \mathrm{O}_{3}$ & 0,12 & - \\
\hline $\mathrm{CaO}$ & 0,26 & $14,96(\mathrm{CaO}+\mathrm{MgO})$ \\
\hline Outros & - & 2,69 \\
\hline Perda ao fogo & 2,16 & 0,28 \\
\hline
\end{tabular}

* Análise por espectroscopia de absorção atômica.

Tabela II - Percentual de RSL introduzido nas composições (\% em massa). STD - formulação padrão (frita).

[Table II - Percent of RSL added to the compositions (weight \%). STD - standard formulation (frit).]

\begin{tabular}{ccc}
\hline Fritas & STD $(\%)$ & RSL $(\%)$ \\
\hline STD & 100 & 0,00 \\
P - 01 & 92,7 & 7,30 \\
P - 02 & 85,1 & 14,90 \\
P - 03 & 75,6 & 24,40 \\
P - 04 & 72,3 & 27,70 \\
P - 05 & 55,3 & 44,70 \\
\hline
\end{tabular}

As composições de fritas foram fundidas em cadinho de mulita a $1500{ }^{\circ} \mathrm{C}$ durante $2 \mathrm{~h}$. Após o término da fusão, o material fundido foi resfriado bruscamente em água, formando-se a frita. Esta foi caracterizada em testes de fusão: o comportamento frente à viscosidade/fluidez das fritas (em fusão) foi aferido em placas inclinadas (em ângulo 


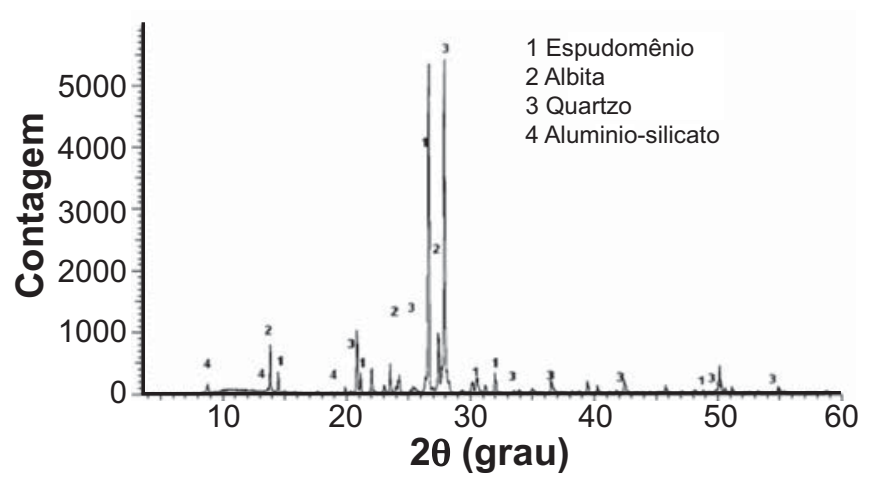

Figura 1: Difratograma de raios X do RSL.

[Figure 1: X-ray diffraction pattern of RSL.]

de $70^{\circ}$ com a superfície) aquecidas simultaneamente com as amostras, as quais preenchiam uma cavidade no topo da placa. Este teste denominado de 'fusibilidade das fritas', é conhecido no jargão industrial como 'teste de botões de fusão ou de escorrimento' e foi realizado na queima a $1150{ }^{\circ} \mathrm{C}$ durante 20 min. A frita produzida anteriormente ('frita de RSL') foi utilizada na confecção de uma formulação de esmalte, a fim de se testar a expansão térmica e de se avaliar os aspectos visuais (transparência, brilho, ausência de bolhas e cristais insolúveis). Essa formulação foi denominada 'esmalte com frita de RSL'. Ela foi preparada do seguinte modo: as amostras de fritas obtidas após a fusão foram pesadas em bateladas de $200 \mathrm{~g}$, conforme a composição apresentada na Tabela II, e submetidas ao processo de moagem via úmido em moinho laboratorial Servitech (CT 242), com tempo de $20 \mathrm{~min}$. Adicionou-se um caulim, um dispersante e um ligante à frita preparada com o RSL (Tabela III). A barbotina resultante apresentou fração granulométrica de 3 a $5 \%$ retida na peneira ABNT de malha 325 (abertura $45 \mu \mathrm{m}$ ) e densidade de $1,76 \mathrm{~g} / \mathrm{cm}^{3}$.

Tabela III - Formulação de esmalte com frita de RSL (\% em massa).

[Table III - Formulation of the glaze with the RSL frit (weight \%).]

\begin{tabular}{lc}
\hline Descrição & Percentual de sólidos (\%) \\
\hline Frita de RSL & 89,70 \\
Caulim & 10,00 \\
CMC & 0,20 \\
HMF & 0,10 \\
Água (\% da massa & 36,00 \\
sólida) & \\
\hline Obs. $H M F$ - hexametafosfato de sódio CMC - carboximetil celulose.
\end{tabular}

As análises termodilatométricas foram realizadas no dilatômetro BP Engenharia (RB 3000-20) com taxa de aquecimento de $5{ }^{\circ} \mathrm{C} / \mathrm{min}$ e temperatura máxima de $1000{ }^{\circ} \mathrm{C}$. Cilindros foram confeccionados em moldes de gesso de $8 \mathrm{~mm}$ de diâmetro e $60 \mathrm{~mm}$ de comprimento, nos quais as suspensões de esmalte foram vazadas, para posterior secagem. Determinou-se o coeficiente de expansão térmica linear e também as temperaturas de transição vítrea $\left(\mathrm{T}_{\mathrm{g}}\right)$, temperatura de amolecimento $\left(\mathrm{T}_{\mathrm{r}}\right)$ e temperatura de acoplamento $\left(\mathrm{T}_{\mathrm{a}}\right)$. Na segunda parte do presente trabalho foi desenvolvido o 'esmalte comercial + RSL', utilizandose as formulações apresentadas na Tabela IV. Objetivou-se comparar o efeito da introdução de teores crescentes do resíduo RSL frente a um esmalte comercial (ES-1). Segundo o fabricante, este esmalte é composto de (percentuais em massa): frita mate $\mathrm{Zn} / \mathrm{Ca}(63 \%)$, alumina $(12 \%)$, caulim $(9,1 \%)$, calcita $(10 \%)$, quartzo $(5 \%)$, hexametafosfato de sódio $(0,7 \%)$ e carboximetil celulose $(0,2 \%)$. A formulação ES-02 refere-se a 95\% do esmalte ES-01 + 5\%RSL.

Tabela IV - Formulações esmalte comercial + RSL (\% em massa).

[Table IV - Formulations of commercial glaze + RSL (weight $\%)$.]

\begin{tabular}{ccc}
\hline Esmalte & ES-01 (\%) & RSL (\%) \\
\hline ES - 01 (padrão comercial) & 100 & 0,0 \\
ES - 02 & 95 & 5,0 \\
ES - 03 & 90 & 10,0 \\
ES - 04 & 85 & 15,0 \\
ES - 05 & 80 & 20,0 \\
ES - 06 & 75 & 25,0 \\
\hline
\end{tabular}

O esmalte foi depositado sobre um suporte cerâmico, com altura de camada inferior a $0,6 \mathrm{~mm}$, nivelado com um aparato de aço (denominado na indústria de binil), cujas dimensões podem ser encontradas em [13]. A queima do 'esmalte com frita de RSL' foi realizada em forno a rolo laboratorial, tendo como combustível o gás natural. $\mathrm{O}$ ciclo térmico utilizado foi de $25 \mathrm{~min}$ e temperatura máxima de $1150{ }^{\circ} \mathrm{C}(12 \mathrm{~min}$ de aquecimento, 6 min de patamar, 7 min de resfriamento). Para o 'esmalte comercial + RSL', a queima foi realizada com ciclo de $42 \mathrm{~min}$ e temperatura máxima de $1200{ }^{\circ} \mathrm{C}(21 \mathrm{~min}$ de aquecimento, 4 min de patamar, 7 min de resfriamento). A avaliação dos aspectos estéticos do esmalte, como cor, brilho e transparência, foi realizada visualmente por técnico experiente (empresa Colorminas - Colorifício e Mineração S.A.), considerando-se a semelhança com a amostra padrão.

\section{RESULTADOS E DISCUSSÃO}

A formulação de frita com o resíduo RSL (Tabela II) foi avaliada primeiramente pela fusão das formulações testadas. O uso dessa frita na formulação de um esmalte (Tabela III) foi avaliado pela expansão térmica e pelos aspectos visuais. A composição de um esmalte com o RSL (Tabela IV) foi estudada pela expansão térmica e pelos aspectos visuais.

\section{Fusibilidade da frita de RSL}

Com a finalidade de se avaliar a fluidez das fritas obtidas, foram realizados os testes de fusibilidade ou 'botões de 
fusão' (queima a $1150{ }^{\circ} \mathrm{C}$ durante $20 \mathrm{~min}$ ). A Fig. 2 apresenta os valores obtidos. Ao se incorporar o RSL, obtêm-se fritas com propriedades de fluidez melhores que a composição de referência, dado pelo comprimento percorrido em placa inclinada pela amostra. Este fator pode ser explicado pela presença do lítio na estrutura das composições com RSL. É bastante conhecido o fato do óxido de lítio reduzir a temperatura de fusão dos silicatos, reduzindo a viscosidade da fase vítrea formada [14, 15]. Esta análise mostra o excelente potencial que o RSL tem como fluxante em auxiliar à vitrificação para diversos produtos da cerâmica tradicional. A adição de óxido de lítio resulta em reação eutética em $\sim 950{ }^{\circ} \mathrm{C}$ no diagrama ternário $\mathrm{SiO}_{2}-\mathrm{Li}_{2} \mathrm{O}-\mathrm{Al}_{2} \mathrm{O}_{3}$, no triângulo de compatibilidade $\mathrm{Li}_{2} \mathrm{O} \cdot 2 \mathrm{SiO}_{2}-\mathrm{SiO}_{2}-\mathrm{Li}_{2} \mathrm{O}$. $\mathrm{Al}_{2} \mathrm{O}_{3} \cdot 4 \mathrm{SiO}_{2}[16]$.

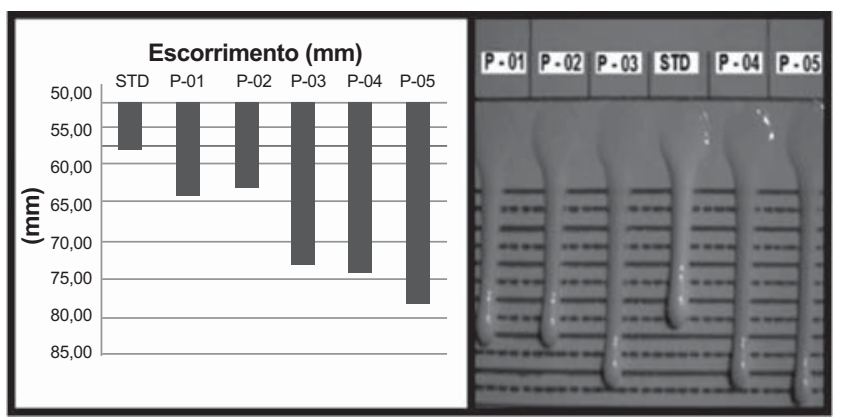

Figura 2: Representação gráfica dos botões de fusão das amostras STD e P - 01 a P - 05.

[Figure 2: Graphical representation of melting buttons of the samples STD and P - 01 to $P$ - 05.]

\section{Análise dilatométrica do esmalte da frita de RSL}

A Tabela $\mathrm{V}$ apresenta os valores do coeficiente de expansão térmica linear, no intervalo de 25 a $325{ }^{\circ} \mathrm{C}$, e também as temperaturas de transição vítrea $\left(\mathrm{T}_{\mathrm{g}}\right)$, temperatura de amolecimento $\left(\mathrm{T}_{\mathrm{r}}\right)$ e temperatura de acoplamento $\left(\mathrm{T}_{\mathrm{a}}\right)$. A Fig. 3 apresenta a curva referente à expansão térmica da formulação P-01. As demais formulações apresentaram

Tabela V - Coeficiente de expansão térmica linear $(\alpha), T_{r^{\prime}}$ $\mathrm{T}_{\mathrm{a}}$ e $\mathrm{T}_{g}$ das composições de esmalte com as fritas obtidas (esmalte da frita de RSL).

[Table $V$-Coefficient of linear thermal expansion ( $\alpha), T_{r}, T_{a}$ and $T_{\mathrm{g}}$ of the glaze compositions of the prepared frits (glaze of RSL̊ frit).]

\begin{tabular}{ccccc}
\hline Frita & $\alpha\left(25-325{ }^{\circ} \mathrm{C}\right)\left(10^{-7}{ }^{\circ} \mathrm{C}^{-1}\right)$ & $\mathrm{T}_{\mathrm{r}}\left({ }^{\circ} \mathrm{C}\right)$ & $\mathrm{T}_{\mathrm{a}}\left({ }^{\circ} \mathrm{C}\right) \mathrm{T}_{\mathrm{g}}\left({ }^{\circ} \mathrm{C}\right)$ \\
\hline STD & 59,40 & 735 & 700 & 669 \\
$\mathrm{P}-01$ & 58,50 & 710 & 682 & 654 \\
$\mathrm{P}-02$ & 57,20 & 711 & 689 & 662 \\
$\mathrm{P}-03$ & 57,70 & 698 & 676 & 650 \\
P- 04 & 56,40 & 708 & 680 & 655 \\
P- 05 & 58,00 & 698 & 673 & 646 \\
\hline STD - Amostra padrão de referéncia. & & &
\end{tabular}

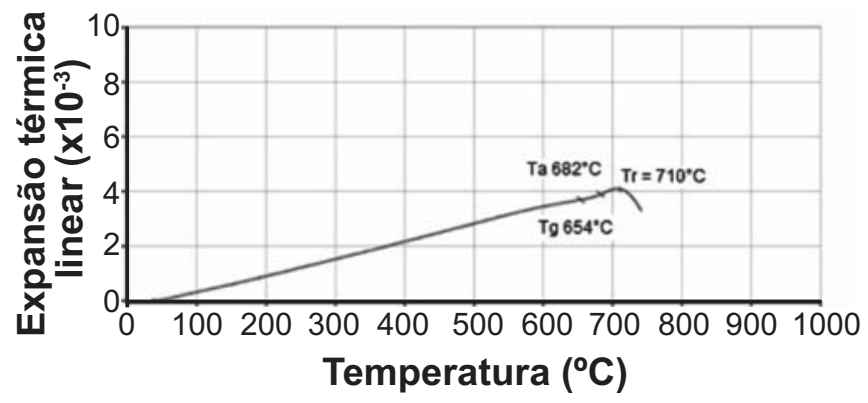

Figura 3: Expansão térmica linear da formulação P-01. Temperaturas de: transição vítrea $\left(\mathrm{T}_{\mathrm{g}}\right)$; amolecimento $\left(\mathrm{T}_{\mathrm{r}}\right)$ e acoplamento $\left(\mathrm{T}_{\mathrm{a}}\right)$.

[Figure 3: Linear thermal expansion of P-01 formulation. Temperatures of: glass transition $\left(T_{g}\right)$; softening $\left(T_{r}\right)$ and coupling $\left.\left(T_{d}\right) \cdot\right]$

comportamento semelhante diante da expansão térmica, de modo que são apresentados somente seus valores (Tabela V).

Comparando-se os valores de coeficiente de expansão térmica linear $(\alpha)$ com o da amostra STD (Tabela V), podese observar uma pequena redução do mesmo devido à introdução do RSL. Os valores aceitáveis (comercialmente) de $\alpha$ encontram-se no intervalo de 57,4 a $61,4 \cdot 10^{-7}{ }^{\circ} \mathrm{C}^{-1}$. Por conseguinte, as amostras P-01, P-03 e P-05 estariam dentro deste limite. No entanto, a redução significativa da temperatura de amolecimento $\left(\mathrm{T}_{\mathrm{r}}\right)$ inviabilizaria o uso das formulações propostas. A temperatura de amolecimento do esmalte deve ser a maior possível, de modo a permitir que todos os gases oriundos da queima do corpo cerâmico sejam eliminados à atmosfera, caso contrário, ficariam retidos no esmalte, gerando defeitos no mesmo [17]. A formulação com melhor resultado (P - 01) apresentou valor de $\mathrm{T}_{\mathrm{r}} 25{ }^{\circ} \mathrm{C}$ abaixo do valor da formulação STD (referência). Os valores de referência estão de acordo com a prática industrial e sua aceitação no mercado.

Como é conhecido, os óxidos modificadores da estrutura vítrea aumentam o coeficiente de expansão térmica linear e reduzem a temperatura do ponto de amolecimento [14]. Assim, em geral, opta-se pela troca de cátions alcalinos (fluxantes) por alcalinos terrosos (estabilizantes). Outra opção é se modificar a quantidade de $\mathrm{B}_{2} \mathrm{O}_{3}$, o qual diminui fortemente o coeficiente de dilatação térmica e a temperatura de fusão [15]. Com base nos dados obtidos, foram realizadas alterações nas composições visando principalmente o aumento (da temperatura) do ponto de amolecimento. Dessa forma, realizaram-se algumas modificações nas formulações e novas medições, encontrando-se o resultado desejado na amostra P-12. Esta foi formulada a partir da amostra

Tabela VI - Coeficiente de expansão térmica linear $(\alpha), \mathrm{T}_{\mathrm{r}}, \mathrm{T}_{\mathrm{a}}$ e $\mathrm{T}_{\mathrm{g}}$ da formulação P-12.

[Table VI - Coefficient of linear thermal expansion ( $\alpha$ ), $T_{r}, T_{a}$ and $T_{g}$ of P-12 formulation.]

\begin{tabular}{ccccc}
\hline Frita & $\alpha\left(25-325{ }^{\circ} \mathrm{C}\right)\left(10^{-7}{ }^{\circ} \mathrm{C}^{-1}\right)$ & $\begin{array}{c}\mathrm{T}_{\mathrm{r}} \\
\left({ }^{\circ} \mathrm{C}\right)\end{array}$ & $\begin{array}{c}\mathrm{T}_{\mathrm{a}} \\
\left({ }^{\circ} \mathrm{C}\right)\end{array}$ & $\begin{array}{c}\mathrm{T}_{\mathrm{g}} \\
\left({ }^{\circ} \mathrm{C}\right)\end{array}$ \\
\hline STD & 59,40 & 735 & 700 & 669 \\
$\mathrm{P}-12$ & 58,70 & 742 & 706 & 681 \\
\hline
\end{tabular}


P-01 (7,30\% de RSL), substituindo-se 1,30\% de óxidos fundentes $\left(\mathrm{Na}_{2} \mathrm{O}+\mathrm{K}_{2} \mathrm{O}\right)$ por $1,30 \%$ de óxidos estabilizantes $(\mathrm{CaO}+\mathrm{MgO})$ e $0,5 \%$ de $\mathrm{B}_{2} \mathrm{O}_{3}$ por $0,5 \%$ de $\mathrm{SiO}_{2}$. A Tabela VI apresenta os valores obtidos após estes ajustes. Este resultado se mostrou coerente com os valores de referência (frita STD- comercial).

\section{Análise visuais do esmalte da frita de RSL}

A Tabela VII apresenta os resultados alcançados através da avaliação visual comparativa das fritas após a queima. A amostra P - 05 alcançou os requisitos estéticos necessários, de modo que ela apresentou parâmetros semelhantes aos da frita de referência, sendo estes igualmente alcançados pela formulação P-12. Os valores da Tabela VII foram avaliados com base na experiência do técnico avaliador [13]. Observase que os materiais desenvolvidos foram aprovados em diversos requisitos, como textura, transparência, brilho e ausência de bolhas e cristais insolúveis.

Tabela VII - Avaliação visual dos esmaltes das fritas RSL obtidas.

[Table VII - Visual evaluation of the glazes obtained with RSL frits.]

\begin{tabular}{cccc}
\hline Frita & Textura & Brilho & Transparência \\
\hline STD & 5 & 5 & 5 \\
P - 01 & 5 & 4 & 5 \\
P - 02 & 4 & 5 & 5 \\
P - 03 & 4 & 6 & 5 \\
P - 04 & 4 & 4 & 4 \\
P - 05 & 5 & 5 & 5 \\
P - 12 & 5 & 5 & 5 \\
\hline
\end{tabular}

Obs: 5: igual a amostra de avaliação (STD); < 5: inferior a amostra de avaliação; > 5: superior a amostra de avaliação.

\section{Análise dilatométrica do esmalte + RSL}

A Tabela VIII apresenta os resultados da análise de expansão térmica linear do esmalte mate de referência (ES-01) e das formulações com as adições de RSL. Podese observar que a introdução do RSL à formulação ES-01 produz uma pequena redução do coeficiente de expansão térmica, sendo $<6 \%$ para a maioria das composições e, portanto, comercialmente aceitável. Essa redução pode ser atribuída à ação fluxante dos óxidos alcalinos presentes no RSL e reação com o quartzo presente na formulação ES-01.

Os esmaltes mate + RSL obtidos após a queima foram submetidos à avaliação visual, a fim de se verificar as variações provenientes do uso do RSL em sua composição. Conforme a Tabela IX, um percentual de até $10 \%$ de RSL (ES-03) pode ser adicionado às composições testadas com manutenção das características desejadas do esmalte de referência, ou seja, pode-se aplicar esses esmaltes sem que ocorra variação significativa das propriedades em um produto
Tabela VIII - Coeficiente de expansão térmica linear dos esmaltes + RSL.

[Table VIII - Coefficient of linear thermal expansion of glazes + RSL.]

\begin{tabular}{cc}
\hline Esmalte & $\alpha\left(25-325^{\circ} \mathrm{C}\right)\left(10^{-7}{ }^{\circ} \mathrm{C}^{-1}\right)$ \\
\hline ES - 01 & 62,80 \\
ES - 02 & 58,80 \\
ES - 03 & 59,20 \\
ES - 04 & 59,30 \\
ES - 05 & 58,80 \\
ES - 06 & 57,80 \\
\hline
\end{tabular}

cerâmico. A partir de $15 \%$ de RSL, tem-se um pequeno aumento de brilho superficial e da intensidade de cor, os quais aumentam ainda mais ao se chegar a $25 \%$ de RSL. Portanto, pode-se optar por utilizar o RSL em percentual de até $10 \%$, mantendo-se as características originais do ES-01, ou em maiores teores de RSL, alterando-se as propriedades estéticas, mas com ganho significativo na intensidade da coloração.

Tabela IX - Resultado da análise visual dos esmaltes mates obtidos.

[Table IX - Results of the visual analysis of the obtained matte glazes.]

Esmalte Textura Brilho Cobertura Desenvolvimento

\begin{tabular}{lllll}
\hline ES - 01 & 5 & 5 & 5 & 5 \\
ES - 02 & 5 & 5 & 5 & 5 \\
ES - 03 & 5 & 5 & 5 & 5 \\
ES - 04 & 5 & 6 & 5 & 6 \\
ES - 05 & 5 & 6 & 4 & 7 \\
ES - 06 & 5 & 7 & 4 & 8 \\
\hline
\end{tabular}

Obs: 5: igual a amostra de avaliação (ES-01); < 5: inferior a amostra de avaliação; > 5: superio a amostra de avaliação.

A Fig. 4 ilustra o incremento na intensidade de cor para o acréscimo de RSL no esmalte. Esta é uma característica muito importante, pois permite o desenvolvimento de esmalte com coloração mais intensa, ou, alternativamente, é possível reduzir a quantidade utilizada dos corantes cerâmicos - um dos componentes de maior custo em uma formulação.

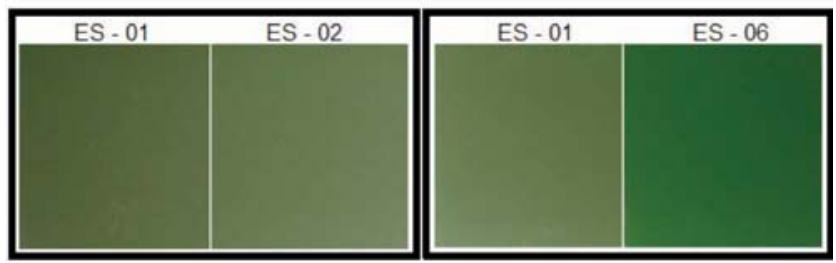

Figura 4: Desenvolvimento de cor esmalte comercial + RSL. [Figure 4: Development of color in commercial glaze + RSL.] 


\section{CONCLUSÃO}

O resíduo do beneficiamento mineral de uma rocha com espodumênio e albita (RSL) pode ser uma interessante matéria-prima para obtenção de esmaltes e fritas cerâmicas. Ele possibilitou a fabricação de produtos, tanto fritas como esmaltes, com características técnicas e estéticas dentro dos padrões utilizados no mercado. A utilização do RSL é uma alternativa interessante para se fornecer óxido de lítio em uma composição, em relação aos minerais ou materiais sintéticos de custo bem mais elevado. $\mathrm{O} \mathrm{Li}_{2} \mathrm{O}$ teve um efeito positivo nas propriedades testadas. A introdução do RSL em uma formulação de frita diminui a temperatura de fusão, determinada pelo aumento da fluidez das amostras testadas em teste de 'botão de fusão'. Isto prova que o RSL apresenta também excelente potencial como fluxante para auxiliar à sinterização de diversos produtos cerâmicos. A incorporação do RSL às fritas produziu a redução do coeficiente de expansão térmica linear e da temperatura de amolecimento, como demonstraram os testes realizados em uma formulação de esmalte. Por meio de ajustes na composição química das formulações, baseando-se na ciência dos vidros, foi possível obter as propriedades almejadas. Especificamente, substituiu-se parte dos óxidos fluxantes $\left(\mathrm{Na}_{2} \mathrm{O}+\mathrm{K}_{2} \mathrm{O}\right)$ por óxidos estabilizantes $(\mathrm{CaO}+\mathrm{MgO})$ e parte do óxido de boro $\left(\mathrm{B}_{2} \mathrm{O}_{3}\right)$ por sílica $\left(\mathrm{SiO}_{2}\right)$, favorecendo o aumento da temperatura de amolecimento e ajustando-se à expansão térmica, sem modificar significativamente outras propriedades. A utilização de até $10 \%$ em massa do RSL em uma formulação de esmalte mate manteve as mesmas propriedades estéticas do esmalte de referência. Em maiores concentrações (10 a $25 \%$ ) a presença de RSL aumenta a intensidade de cor sem alterar outros parâmetros. Salientase que, como os corantes estão dentre as matérias-primas de maiores custos utilizadas pela indústria cerâmica, o uso do RSL torna-se bastante atrativo, pois permite a redução no consumo de corantes, o que por sua vez contribui para a redução de custos pela indústria cerâmica.

\section{REFERÊNCIAS}

[1] T.L. Zanin, W. Klitzke, L.F.L. Luz Jr., Cerâmica 59, 350 (2013) 231-234.

[2] A.M.G.D. Mendonça, J.M. Cartaxo, R.R. Menezes, L.N.L. Santana, G.A. Neves, H.C. Ferreira, Cerâmica 58, 346 (2012) 216-224.

[3] T. Tarvornpanich, G.P. Souza, W.E. Lee, J. Am. Ceram. Soc. 88, 5 (2005) 1302-1308.

[4] L.P. Garcia, R.T. da Cruz, S.R. Bragança, Proc. IMech E Part L: J. Mater. Des. Appl. 226, 4 (2012) 286-292.

[5] L.A. Carus, S.R. Bragança, Mater. Res. 16, 6 (2013) 13981404.

[6] R.C. da Silva, S.A. Pianaro, S.M. Tebcherani, Ceram. Int. 38 (2012) 2725-2731.

[7] E.C.S. N Serpa, Diss. Mestrado, Pós-ENQ, UFSC (2007) $67 \mathrm{p}$.

[8] M. Oberzan, J. Holc, M. Buhb, D. Kuscer, I. Lavrac, M. Kosec, J. Eur. Cer. Soc. 29 (2009) 2143-2152.

[9] A. Tucci, L. Esposito, E. Rastelli, C. Palmonari, E. Rambaldi, J. Eur. Ceram. Soc. 24 (2004) 83-92.

[10] S.R. Bragança, H.C. M. Lengler, C.P. Bergmann, Cerâmica 56 (2010) 297-299.

[11] C.W. Sinton, Raw materials for glass and ceramics, John Wiley \& Sons, New Jersey (2006).

[12] H.C.M. Lengler, Tese Dr., PPGEM-UFRGS (2006) 177p.

[13] J.L. Coelho, Diss. Mestrado, PPGEM-UFRGS (2014) $118 \mathrm{p}$.

[14] J.M.F. Navarro, El vidrio, 2 Ed., Consejo Superior de Investigaciones Científicas - Instituto de Cerámica y Vidrio, Madrid (1991).

[15] W.E. Matthes, Vidriados cerámicos, Ed. Omega, Barcelona (1990).

[16] R.A. Eppler, J. Am. Ceram. Soc. 46, 2 (1963) 100.

[17] Associazione Contruttori Italiani Macchine Attrezzature per Ceramica (ACIMAC), La smaltatura e decorazione delle piastrelle ceramiche, Ed. SALA, Modena (2000).

(Rec. 28/08/2015, Rev. 29/09/2015, 02/10/2015, Ac. 07/10/2015) 